Vittalle - Revista de Ciências da Saúde v. 31, n. 2 (2019) 10-16
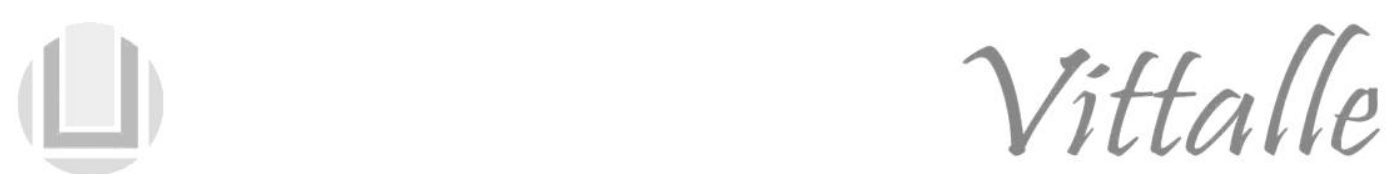

\title{
Associação entre o perfil antropométrico inicial e o desfecho de ganho ponderal em gestantes
}

\author{
Marilía Bandeira Muniz , Eliziane Costa da Silva ${ }^{\mathrm{b}}$, Samanta Siqueira de Almeida ${ }^{\mathrm{b}}$, \\ Danielle Erilane Silva Pereira ${ }^{b}$, Giselle de Paula da Silva ${ }^{c}$, Caroline Neves de Morais ${ }^{a}$, \\ Dayse Rafaele Lima dos Santos Silva ${ }^{\mathrm{b}}$, Elda Silva Augusto de Andrade ${ }^{\mathrm{b} *}$.
}

${ }^{a}$ Faculdade Pernambucana de Saúde -FPS, Recife, PE, Brasil

${ }^{\mathrm{b}}$ Instituto de Medicina Integral Professor Fernando Figueira - IMIP, Recife, PE, Brasil

${ }^{c}$ HospitalReal Hospital Português de Beneficência do Recife, Recife, PE, Brasil

\section{Histórico do Artigo \\ Recebido em \\ 26/03/2019 \\ Aceito em \\ 08/08/2019}

Palavras-chave:

Gestação; índice de massa corporal; avaliação nutricional

Keywords:

Gestation; body mass index; nutritional assessment

\begin{abstract}
RESUMO
O presente trabalho teve como objetivo avaliar a associação entre o perfil antropométrico inicial e o desfecho de ganho ponderal em gestantes. Estudo longitudinal em gestantes atendidas no ambulatório de nutrição infantil do Instituto de Medicina Integral Professor Fernando Figueira, no período de junho a setembro de 2015. Os dados antropométricos foram coletados na primeira consulta do pré-natal e na última que antecedeu o parto. O parâmetro utilizado para o diagnóstico nutricional foi o Índice de Massa Corporal. As análises foram realizadas no Programa Statistical Package for the Social Sciences versão 22.0 e realizadas testes de associação de variáveis. Foram avaliadas 69 gestantes com média de idade de 26 anos. No período pré-gestacional inicial, 52,17\% das mulheres apresentavam sobrepeso ou obesidade. No período gravídico, o ganho de peso elevado foi encontrado em 44,93\%. Houve associação entre o excesso de peso no período prégestacional e o ganho de peso elevado. A avaliação do estado nutricional mostrou que mais de 50\% das mulheres já iniciaram a gestação com sobrepeso/obesidade, sendo semelhanteao encontradoem um estudo retrospectivo no município de São Paulo e em um estudo descritivo realizado na cidade do Recife. A maioria das gestantes apresentou inadequação do ganho de peso gestacional, evidenciando a importância da promoção de mudança de estilo de vida, sendo necessário o acompanhamento nutricional desde o período pré-gestacional visando evitar complicações futuras para o binômio mãe e filho.
\end{abstract}

\section{Association between initial anthropometric profile and performance gain performance in pregnant women}

\begin{abstract}
The present study aimed to evaluate the association between the initial anthropometric profile and the weight gain outcome in pregnant women. Longitudinal study of pregnant women attended at the Infant Nutrition Outpatient Clinic of the Instituto de Medicina Integral Professor Fernando Figueira from June to September, 2015. Anthropometric data was collected at the first prenatal visit and at the last prenatal visit. The parameter used for the nutritional diagnosis was the Body Mass Index. The analyzes were carried out in the Program Statistical Package for the Social Sciences version 22.0 and carried out tests of association of variables. A total of 69 pregnant women with mean age of 26 years were evaluated. In the initial pre-gestational period, $52.17 \%$ of the women were overweight or obese. In the pregnancy period, the high weight gain was found in $44.93 \%$. There was an association between pre-gestational weight gain and high weight gain. The evaluation of the nutritional status showed that more than $50 \%$ of the women have already begun pregnancy with overweight / obesity, similar to that found in a retrospective study in the city of São Paulo and in a descriptive study carried out in the city of Recife. The majority of pregnant women showed inadequate gestational weight gain, evidencing the importance of lifestyle change promotion, and it is necessary nutritional monitoring from the pre gestational period in order to avoid future complications for the mother and child binomial.
\end{abstract}

\footnotetext{
* Autor correspondente: elda.saa@gmail.com (Andrade E.S.A.)
} 


\section{Introdução}

A gestação é um período de intensas alterações físicas, hormonais e emocionais, nas quais as necessidades nutricionais e ingestão da mãe modificam-se completamente. $\mathrm{O}$ estado nutricional no início da gestação e o acompanhamento do ganho de peso são de suma importância, uma vez que estão diretamente relacionados ao risco de morbimortalidade materna e perinatal (1). O ganho de peso tanto insuficiente como em excesso, apresentam situações desfavoráveis para mãe e filho, dentre elas; parto cirúrgico, diabetes e hipertensão gestacional, baixo peso ao nascer, macrossomia fetal, desproporção céfalo-pélvica, trauma, asfixia, prematuridade e morte perinatal (2). Essas complicações perinatais associadas à inadequação nutricional, pode promover enfermidades a vida adulta (3).

Segundo a Organização Mundial da Saúde (OMS), o monitoramento do ganho ponderal durante a gestação é um procedimento de baixo custo e de grande utilidade para o estabelecimento de intervenções nutricionais, visando à redução de riscos maternos e fetais. A avaliação e a orientação nutricional podem proporcionar um ganho de peso adequado, prevenindo o ganho excessivo ou diagnosticando o ganho de peso insuficiente (4).

As recomendações para ganho de peso total segundo o estado nutricional inicial da gestante são classificadas de acordo com o Ministério da Saúde (MS), através do índice de massa corporal (IMC) por semana gestacional (1). Através destes parâmetros é possível proporcionar a gestantes o ganho de peso necessário para suprir as demandas materno-fetal, melhorando assim o desfecho nutricional e atendendo desta forma, os programas da atenção básica relacionados à saúde (5).

Desta forma, a atenção básica na fase pré-natal, tem o intuito de realizar o acompanhamento e desenvolvimento gestacional, através de ações de prevenção e promoção da saúde, diagnóstico precoce e tratamento adequado de qualquer problema que venha a ocorrer durante o período gestacional (5).

Nesse contexto, a avaliação do ganho ponderal ao longo da gestação e a compreensão de seus determinantes são de grande relevância para o estabelecimento de intervenções precoces, visando assegurar a saúde materno-infantil (6). Com isso, o objetivo deste estudo foi avaliar a associação entre operfil antropométrico inicial e o desfecho de ganho ponderal em gestante.

\section{Materiais e métodos}

Trata-se de um estudo do tipo longitudinal, realizado com gestantes no período de junho a setembro de 2015, no ambulatório de nutrição infantil do Instituto de Medicina Integral Prof. Fernando Figueira - IMIP (Recife-PE), hospital de referência materno infantil. Amostra não probabilística por conveniência sendo eleitos para este estudo gestantes com faixa etária de 18 a 39 anos, totalizando o valor de 69 indivíduos. Foram excluídos deste estudo, pacientes que apresentavam doenças de ordem genéticas ou metabólicas que pudessem repercutir no seu estado nutricional, tais como doenças crônicas (doença renal crônica, doenças hepáticas crônicas e diabetes).

Foram coletados dados antropométricos de peso pré-gestacional ou peso real realizado até a $13^{\mathrm{a}}$ semana de gestação e altura. A altura foi aferida em posição ortostática utilizando o antropômetro vertical acoplado a balança digital - Filizola® com a gestante de pés descalços e unidos, no centro da plataforma, de costas para o marcador. $\mathrm{O}$ peso foi aferido na balança (Filizola ${ }^{\circledR}$ ), que apresentava precisão de $0,5 \mathrm{Kg}$ e capacidade de $150,0 \mathrm{Kg}$. As pacientes foram pesadas descalças e com o mínimo de vestimentas. 
Posteriormente, foi calculado o IMC, tendo como variáveis o peso sobre a altura ao quadrado. Utilizou-se como parâmetro para o diagnóstico nutricional pré-gestacional o IMC, tendo como ponto de corte as referências de baixo peso (IMC $<18,5 \mathrm{~kg} / \mathrm{m}^{2}$ ), peso adequado $\left(\geq 18,5 \mathrm{~kg} / \mathrm{m}^{2} \leq 24,9 \mathrm{~kg} / \mathrm{m}^{2}\right)$, sobrepeso $\left(\geq 25 \mathrm{~kg} / \mathrm{m}^{2} \leq 29,9 \mathrm{~kg} / \mathrm{m}^{2}\right)$ e obesidade $\left(\geq 30 \mathrm{~kg} / \mathrm{m}^{2}\right)(7)$. O estado nutricional durante a gestação foi classificado de acordo com os valores do IMC para a idade gestacional, proposto pelo método de Atalah et al. (8). Os dados antropométricos foram coletados em dois momentos, na primeira consulta do pré-natal e na última consulta que antecedeu o parto.

O presente estudo foi aprovado pelo Comitê de Ética em Pesquisa do IMIP, sob o $\mathrm{n}^{\circ}$ CAAE 39934814.9.0000.5201, de acordo com a Resolução ${ }^{\circ}$ 466/12 do Conselho Nacional de Saúde.

Os dados foram digitados no programa Excel para Windows®. As análises foram realizadas no Programa SPSS versão 22.0. As variáveis contínuas foram testadas quanto à normalidade da distribuição, pelo teste de Kolmogorov Smirnof e aplicadas transformações logarítmicas $(\log n$ ), já variáveis com distribuição normal foram descritas sob a forma de médias e dos respectivos desvios padrão. As variáveis com distribuição não Gaussiana foram apresentadas sob a forma de medianas e dos respectivos intervalos interquartílicos. Na descrição das proporções, a distribuição binomial foi aproximada à distribuição normal, pelo intervalo de confiança de $95 \%$. Nos testes de inferência estatística, as proporções foram associadas pela razão sendo aplicado o teste p-valor $(\chi 2)$. Foi adotado o nível de significância de 5\% para rejeição de hipótese denulidade.

\section{Resultados}

No total da amostra foram avaliadas 69 gestantes, com média de idade de 26,1 anos \pm 5,57anos, sendo a maioria primíparas. Em relação ao perfil sócio econômico, a média de número de pessoas na família foi de $3,27 \pm 1,49$, onde a maioria das gestantes apresentava uma renda familiar $\leq$ a 2 salários mínimos com um per capta de $\mathrm{R} \$ 514,64$ e tempo de estudo $\geq$ a 12 anos $(86,95 \%)$.

Em relação a assistência pré-natal, o número médio de consultas foi de 7,7 $\pm 2,06$. Quanto ao acompanhamento nutricional das gestantes, a maioria recebeu assistência a partir do $2^{\circ}$ trimestre gestacional $(59,4 \%)$ (Tabela 1$)$. Verificou- se que a maioria das participantes $(52,2 \%)$, iniciaram a gravidez com o estado nutricional inadequado, predominando o sobrepeso/obesidade no período pré- gestacional e mantiveram um ganho ponderal elevado durante todo o período gravídico (Tabela 2), com uma média de idade gestacional de 38,69 semanas $\pm 1,81$ semanas. Com isso, conclui-se que as gestantes que apresentaram sobrepeso no período pré-gestacional apresentaram ganho ponderal maior que as eutróficas e obesas, e esse ganho foi estatisticamente significante $(\mathrm{p}=0,026)$ (Tabela 3).

Tabela1 - Distribuição percentual da paridade materna e do período inicial do acompanhamento nutricional em gestantes atendidas no ambulatório de nutrição de um Hospital de Referência do Nordeste Brasileiro

\begin{tabular}{|ccc}
\hline VARIÁVEIS & N & $\%$ \\
\hline Paridade materna & & \\
Primípara & 43 & 62,31 \\
$\mathbf{2}^{\mathbf{0}}$ gestação & 18 & 26,09 \\
$\mathbf{3}^{\mathbf{0}}$ gestação & 8 & 11,59 \\
Período inicial do acompanhamento & & \\
nutricional & 9 & 13,04 \\
$\mathbf{1}^{\mathbf{0}}$ trimestre & 41 & 59,42 \\
$\mathbf{2}^{\mathbf{0}}$ trimestre & 19 & 27,54 \\
$\mathbf{3}^{\mathbf{0}}$ trimestre & & \\
\hline
\end{tabular}


Tabela 2 - Estado nutricional pré-gestacional e ganho ponderal de gestantes atendidas em um Hospital de Referência do Nordeste, Brasil.

\begin{tabular}{ccc}
\hline VARIÁVEIS & N & \% \\
\hline Estado nutricional pré- gestacional & & 47,82 \\
Eutrófico & 33 & 34,78 \\
Sobrepeso & 24 & 17,39 \\
Obeso & 12 & 23,19 \\
Ganho ponderal total & & 31,88 \\
Baixo & 16 & 44,93 \\
Adequada & 22 & 31 \\
Elevado
\end{tabular}

Tabela 3 - Associação entre o Estado nutricional pré-gestacional e o desfecho do ganho ponderal de gestantes atendidas em um Hospital de Referência do Nordeste, Brasil.

\begin{tabular}{cccc}
\hline \multirow{2}{*}{ GANHO PONDERAL } & \multicolumn{4}{c}{ ESTADO NUTRICIONAL } \\
\cline { 2 - 4 } Baixo & Eutrófico & Sobrepeso & Obesidade \\
Normal & $81,3 \%$ & $6,3 \%$ & $12,5 \%$ \\
Elevado & $45,5 \%$ & $36,4 \%$ & $18,2 \%$ \\
Pearson Chi-Square & $32,3 \%$ & $48,4 \%$ & $19,4 \%$ \\
\hline
\end{tabular}

\section{Discussão}

A maioria das gestantes eram primíparas, com renda familiar mensal inferior ou igual a um e meio salário mínimo, renda per capta de $\mathrm{R} \$ 514,64$ e com mais de 4 anos de estudo. A literatura mostra que as condições socioeconômicas desfavoráveis, repercutem negativamente na saúde da população, e que quanto maior a renda, maior o acesso à alimentação variada (9). Konno et al. mostram que as gestantes de maior escolaridade também obtiveram maior ganho ponderal (10), assim como Hickey que em uma revisão de literatura, conclui que existe uma relação direta entre a escolaridade e o ganho de peso (11), corroborando desta forma com o nosso estudo. Já o estudo realizado em Recife mostra que as gestantes com baixa escolaridade tiveram um aumento de ganho ponderal, uma vez que as mesmas priorizavam o consumo de alimentos ricos em gorduras saturadas, açúcar e alimentos refinados, em substituição a uma alimentação saudável (12), provavelmente por esses alimentos apresentarem um custo menor, além da associação com a falta de informação nutricional, já que o acompanhamento nutricional não foi iniciado no período pré-gestacional.

O tempo de início do acompanhamento nutricional foi um dos fatores limitantes deste estudo, visto que o mesmo só foi iniciado a partir do segundo trimestre. Um estudo transversal com 240 gestantes em Belo Horizonte relata que o acompanhamento nutricional deve ser iniciado desde o período pré-gestacional (13). Os achados do estudo de Santos et al. ressaltam que o controle de peso materno será mais eficaz quando iniciado durante o período pré-concepcional, assegurando assim, condições adequadas a futura gestante e ao seu concepto (6). Recentemente, Fraser et al. investigaram a associação entre ganho de peso gestacional e desfechos gestacionais 16 anos após a gravidez e descobriram que as mulheres com ganho de peso adequado durante a gravidez tiveram melhores resultados em comparação com aquelas que tiveram ganho de peso excessivo (14), que corrobora com o estudo de Padilha et al., que mostra que a redução de complicações fortalece a importância do modelo de atenção nutricional com seu número mínimo de visitas, assistência nutricional precoce e individualizada (15). Com isso conclui-se que o excesso de peso alcançado pelas gestantes desse estudo, pode estar associado ao acompanhamento nutricional tardio. Assim, o monitoramento da avaliação nutricional é de extrema importância, pois influenciará de maneira direta na mudança de hábitos alimentares. 
A avaliação do estado nutricional mostrou que mais de $50 \%$ das mulheres já iniciaram a gestação com sobrepeso/obesidade, sendo semelhanteao encontrado em um estudo retrospectivo no município de São Paulo (5). Um estudo descritivo com 240 gestantes de baixo risco obstétrico, atendidas em um serviço público de pré- natal da cidade do Recife, PE, verificou que $26 \%$ apresentavam sobrepeso/obesidade inicial, que se associou ao ganho de peso excessivo (12). No Brasil, resultados semelhantes foram encontrados em estudo de coorte coordenado pelo Núcleo de Pesquisa em Nutrição e Saúde da Universidade de São Paulo (NUPENS/USP), com dados de 115 gestantes que iniciaram pré-natal no Programa Saúde da Família (PSF) do município de Campina Grande-PB, no qual 27\% apresentavam-se com sobrepeso/obesidade (16). A semelhança desses achados em diferentes regiões do Brasil, indica que no Nordeste, a obesidade, e não só a desnutrição, corrobora com a transição epidemiológica e nutricional do país, onde visualizamos o declínio da desnutrição e a ascensão da obesidade (6).

O estudo multicêntrico dos Estados Unidos com 2.070 gestantes acompanhadas durante 10 anos, mostra que mulheres com sobrepeso no início da gestação ganharam mais peso em relação às eutróficas (17). Isso é preocupante, uma vez que a obesidade materna está associada a complicações tanto para a mãe como para o filho e com isso, comprova-se a importância do monitoramento nutricional dessas gestantes desde o período pré-gestacional, através de intervenções pautadas em orientações dietéticas. Provavelmente, essas gestantes não possuem hábitos alimentares saudáveis e são sedentárias, contribuindodessa forma para o ganho excessivo de peso.

Esse ganho de peso excessivo está presente em 44,9\% das gestantes do nosso estudo, o que também comprovou o estudo randomizado em um município de Porto Alegre (18). Um estudo desenvolvido em seis capitais brasileiras verificou que $29 \%$ das gestantes apresentavam ganho de peso excessivo, proporção queaumentou para 50\% entre aquelas com sobrepeso e $38 \%$ para aquelas com obesidade (6). Esses dados corroboram com nosso estudo, onde as gestantes com sobrepeso no período pré-gestacional, continuaram ganhando mais peso em relação às eutróficas e as obesas (Tabela 3). Esses resultados podem estar associados ao maior risco de hipertensão, diabetes gestacional, préeclâmpsia, morte fetal, macrossomia, parto prematuro e mortalidade perinatal6. A obesidade é uma enfermidade multifatorial, que atinge mulheres na faixa etária reprodutiva na proporção de 6,9\% (18-24 anos), 12,4\% (25-34 anos) e de 17,1\% (35-44 anos). $\mathrm{O}$ excesso de peso, no Brasil, relaciona-se com o pobre perfil nutricional, devido às condições ambientais e socioeconômicas desfavoráveis (19).

O ganho de peso gestacional e o estado nutricional materno vêm se caracterizando como um problema de saúde pública, sendo necessário intervenção imediata nos sistemas de saúde, a fim de promover prevenção de intercorrências gestacionais e influência nas condições de saúde do binômio mãe/filho (20-22). Essa intervenção deve voltar-se para um atendimento nutricional individualizado por meio da avaliação do estado nutricional da gestante nas consultas pré-natal, para que sejam estabelecidas as necessidades de nutrientes necessárias nesse período (6).

\section{Conclusão}

O estudo revela um percentual significativo de puérperas com inadequação do ganho de peso gestacional, evidenciando assim a importância da promoção de mudança de estilo de vida, sendo necessárias intervenções em relação ao estado nutricional prégestacional, ganho de peso gestacional e adequação do consumo alimentar. No entanto trás como fator limitante o tamanho da amostra, sendo necessários mais estudos que contemple uma população maior, captando mulheres em período pré-gestacional ou no 
primeiro trimestre de gestação para que este acompanhamento seja melhor observado.

Com isso, conclui-se a importância da atuação da equipe multiprofissional, que vem com o intuito de encaminhar essa gestante o mais precoce possível para uma avaliação nutricional. Desta forma, os nutricionistas devem estar preparados para orientar as mulheres da importância da redução do peso antes do início da gravidez e também de como evitar o excesso de ganho de peso na gestação. Para que isto seja realizado, a avaliação nutricional deve ser iniciada desde o período pré-gestacional.

\section{Referências}

1. Ministério da Saúde (BR). Secretaria de Atenção Básica à Saúde. Departamento de Ações Programáticas Estratégicas. Área Técnica de Saúde da Mulher. Gestação de alto risco - manual técnico. 2012, Brasília: Ministério daSaúde.

2. Crane JM, white J, Murphy P, Burrage L, Hutchens D. The effect of gestacional weight gain by body mass index on maternal and neonatal outcomes. J obstetGynaecol Can 2009; 31(1):28-35.

3. Zadick Z. Maternal nutrition, fetal weight, body composition and disease in later life. J Endocrinol Invest 2003;26:941-6.

4. Gonçalves CV, Mendoza-Sassi RA, Cesar JA, Castro NB, Bortolomedi AP. Índice de massa corporal e ganho de peso gestacional como fatores preditores de complicações e do desfecho da gravidez. Rev. Bras Ginocol Obstet 2012; 34: 304-9.

5. SatoAPS,FujimoriE.Estadonutricionaleganhodepesodegestantes.Rev.Latino-Am. Enfermagem 2012; 20(3):[7 telas].

6. Santos JGC, Silva JMC, Passos AMPR, Monteiro BKSM, Maia MM et al. Peso materno em gestantes de baixo risco na atenção pré- natal. Internacional Journal of Nutrology 2017;10(2):5-15.

7. World Health Organization [Internet]. Global database on body mass index: an interactive surveillance tool for monitoring nutrition transition. 2012 [cited 2012 May 10]. Available from: <http://apps.who.int/bmi/index.jsp>

8. Atalah SE, Castillo CL, Castro RS. Propuesta de um nuevo estandar de evaluacion nutricional em embarazadas. RevMed Chile 1997; 125:1429-36.

9. Marano D, Gama SGN, Pereira APE, Junior PRBS. Adequação do ganho de peso ponderal de gestantes em dois municípios do estado do Rio de Janeiro (RJ), Brasil. Rev Bras Ginecol Obstet 2012;34(8):386-93.

10. Konno SC, Benicio MHA, Barros AJD. Fatores associados à evolução ponderal de gestantes: uma análise multinível. Rev Saúde Pública 2007; 41(6): 995-1002.

11. Hickey CA. Sociocultural and behavior al influences on weight gain during pregnancy. Am J ClinNutr 2000; 71(5Supl):1364S-70S.

12. Andreto LM, Souza AI, Figueiroa JN, Cabral-Filho JE. Fatores associados ao ganho ponderal excessivo em gestantes atendidas em um serviço público de pré- natal na cidade de Recife, Pernambuco, Brasil. Cad Saúde Pública 2006; 22(11):2401-9.

13. Teixeira CSS, Cabral ACV. Avaliação nutricional de gestantes sob acompanhamento em serviços de pré-natal distintos: a região metropolitana e o ambiente rural. Rev Bras Ginec Obst 2016;38:27-34.

14. Fraser A, Tilling K, Macdonald-Wallis C, Hughes R, Sattar N, Nelson SM et al. Associations of gestational weight gain with maternal body mass index, waist circumference, and blood pressure measured 16 y after pregnancy: the Avon longitudinal Study of Parents and Children (ALSPAC). Am J Clin Nutr 2011; 93:1285-92.

15. Padilha PC, Larissa MO, Neves EQC, Ghedini AC,Costa2 T, Saunders C. Evaluation of efficacy and effectiveness of prenatal nutritional care on perinatal outcome of pregnant women; Rio de Janeiro, Brazil. Nutr Hosp 2015;32(2):845-854.

16. Mello AS, Assunção PL, Gondim SS, Carvalho DF, Amorim MM, Benicio MH, et al. Estado nutricional materno, ganho de peso gestacional e peso ao nascer. Rev Bras Epidemiol 2007; 10(2): 249-57.

17. Gunderson EP, Murtaugh MA, Lewis CE, Quesenberry CP, West DS, Sidney S. Excess gains in 
weight and waist circumference associated with childbearing: the coronary artery risk development in young adults study (CARDIA). Int J Obes Relat Metab Disord2004;28(4):525-35.

18. Nast M, Oliveira A, Rauber F, Vitolo MR. Ganho de peso excessivo na gestação é fator de risco para o excesso de peso em mulheres. Rev Bras Ginecol Obstet 2013;35(12):536-40.

19. Correia LL, Silveira DMI, Silva AC, Campos JS, Machado MMT, Rocha HAL, et al. Prevalência e determinantes de obesidade e sobrepeso em mulheres em idade reprodutiva residentes na região semiárida do Brasil. Ciência \& Saúde Coletiva 2011;16(1):133-45.

20. Dodd JM, Turnbull DA, Mcphee AJ, Wittert G, Crowther CA, Robinson JS. Limiting weight gain in overweight and obese women during pregnancy to improve health outcomes: the LIMIT randomised controlled trial. BMC Pregnancy Childbirth 2011;11:79.

21. Seabra G, Padilha PC, Queiroz JA, Saunders C. Pregestational overweight and obesity: prevalence and outcome associated with pregnancy. Rev Bras Ginecol Obstet 2011; 33(11):348-53.

22. Seligman LC, Duncan BB, Branchtein L, Gaio DS, Mengue SS, Schmidt MI. Obesity and gestational weight gain: cesarean delivery and labor complications. Rev Saúde Pública 2006; 40(3):457-65. 